\title{
Fine-pitch and thick-foil gas electron multipliers for cosmic x-ray polarimeters
}

Toru Tamagawa, Asami Hayato, Yorito Yamaguchi, Hideki Hamagaki, Shigehira Hashimoto, et al.

Toru Tamagawa, Asami Hayato, Yorito Yamaguchi, Hideki Hamagaki, Shigehira Hashimoto, Masahide Inuzuka, Hiromasa Miyasaka, Ikuya Sakurai, Fuyuki Tokanai, Kazuo Makishima, "Fine-pitch and thick-foil gas electron multipliers for cosmic x-ray polarimeters," Proc. SPIE 6266, Space Telescopes and Instrumentation II: Ultraviolet to Gamma Ray, 62663W (15 June 2006); doi: 10.1117/12.671244

Event: SPIE Astronomical Telescopes + Instrumentation, 2006, Orlando, Florida , United States 


\title{
Fine-pitch and thick-foil gas electron multipliers for cosmic x-ray polarimeters
}

\author{
Toru Tamagawa ${ }^{a, b}$, Asami Hayato ${ }^{b, a}$, Yorito Yamaguchi ${ }^{c}$ Hideki Hamagaki $^{c}$, \\ Shigehira Hashimoto ${ }^{b, a}$, Masahide Inuzuka ${ }^{d}$, Hiromasa Miyasaka ${ }^{e}$, Ikuya Sakurai ${ }^{f}$, \\ Fuyuki Tokanai $^{g}$, Kazuo Makishima ${ }^{h, a}$ \\ ${ }^{a}$ RIKEN, The Institute of Physical and Chemical Research, 2-1 Hirosawa, Wako, \\ Saitama 351-0198, Japan; \\ ${ }^{b}$ Department of Physics, Tokyo University of Science, 1-3 Kagurazaka, \\ Shinjyuku-ku, Tokyo 162-8601, Japan; \\ ${ }^{c}$ Center for Nuclear Study (CNS), University of Tokyo, 2-1 Hirosawa, Wako, \\ Saitama 351-0198, Japan; \\ ${ }^{d}$ National Research Institute for Cultural Properties, Tokyo, 13-43 Ueno Park, \\ Taito-ku, Tokyo 110-8713, Japan; \\ ${ }^{e}$ Caltech, MC 220-47, Pasadena, CA 91125, USA;

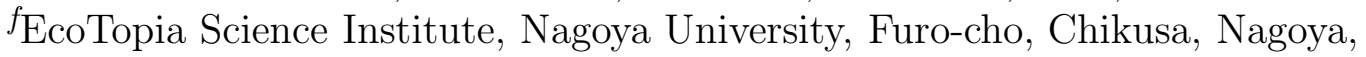 \\ Aiti 464-8603, Japan; \\ ${ }^{g}$ Department of Physics, Yamagata University, 1-4-12 Kojirakawa, \\ Yamagata 990-8560, Japan; \\ ${ }^{h}$ Department of Physics, University of Tokyo, 7-3-1 Hongo, Bunkyo-ku, \\ Tokyo 113-0033, Japan
}

\begin{abstract}
We have produced various gas electron multiplier foils (GEMs) by using laser etching technique for cosmic X-ray polarimeters. The finest structure GEM we have fabricated has $30 \mu \mathrm{m}$-diameter holes on a $50 \mu \mathrm{m}$-pitch. The effective gain of the GEM reaches around 5000 at the voltage of $570 \mathrm{~V}$ between electrodes. The gain is slightly higher than that of the CERN standard GEM with $70 \mu \mathrm{m}$-diameter holes on a $140 \mu \mathrm{m}$-pitch. We have fabricated GEMs with thickness of $100 \mu \mathrm{m}$ which has two times thicker than the standard GEM. The effective gain of the thick-foil GEM is $10^{4}$ at the applied voltage of $350 \mathrm{~V}$ per $50 \mu \mathrm{m}$ of thickness. The gain is about two orders higher than that of the standard GEM. The remarkable characteristic of the thick-foil GEM is that the effective gain at the beginning of micro-discharge is quite improved. For fabricating the thick-foil GEMs, we have employed new material, liquid crystal polymer (LCP) which has little moisture absorption rate, as an insulator layer instead of polyimide. One of the thick-foil GEM we have fabricated has $8 \mu \mathrm{m}$ copper layer in the middle of the 100 $\mu \mathrm{m}$-thick insulator layer. The metal layer in the middle of the foil works as a field-shaper in the multiplication channels, though it slightly decreases the effective gain.
\end{abstract}

Keywords: Cosmic X-ray Polarimeter, gas electron multiplier, GEM, proportional counter

\section{INTRODUCTION}

The gas electron multiplier (GEM) foil has been developed at CERN since the pioneering work by Sauli in $1996{ }^{1}$ The basic structure of the GEM is that small, densely packed holes are drilled through copper-plated polyimide. When a potential difference is applied between the two copper electrodes in a suitable gas, the foil works as an electron multiplier of a proportional counter. Recently, GEM foils have been used in many applications, such as particle tracking, ${ }^{2}$ photon detectors, ${ }^{3-5}$ X-ray imagers. ${ }^{6}$

Further author information: (Send correspondence to Toru Tamagawa) E-mail: tamagawa@riken.jp 

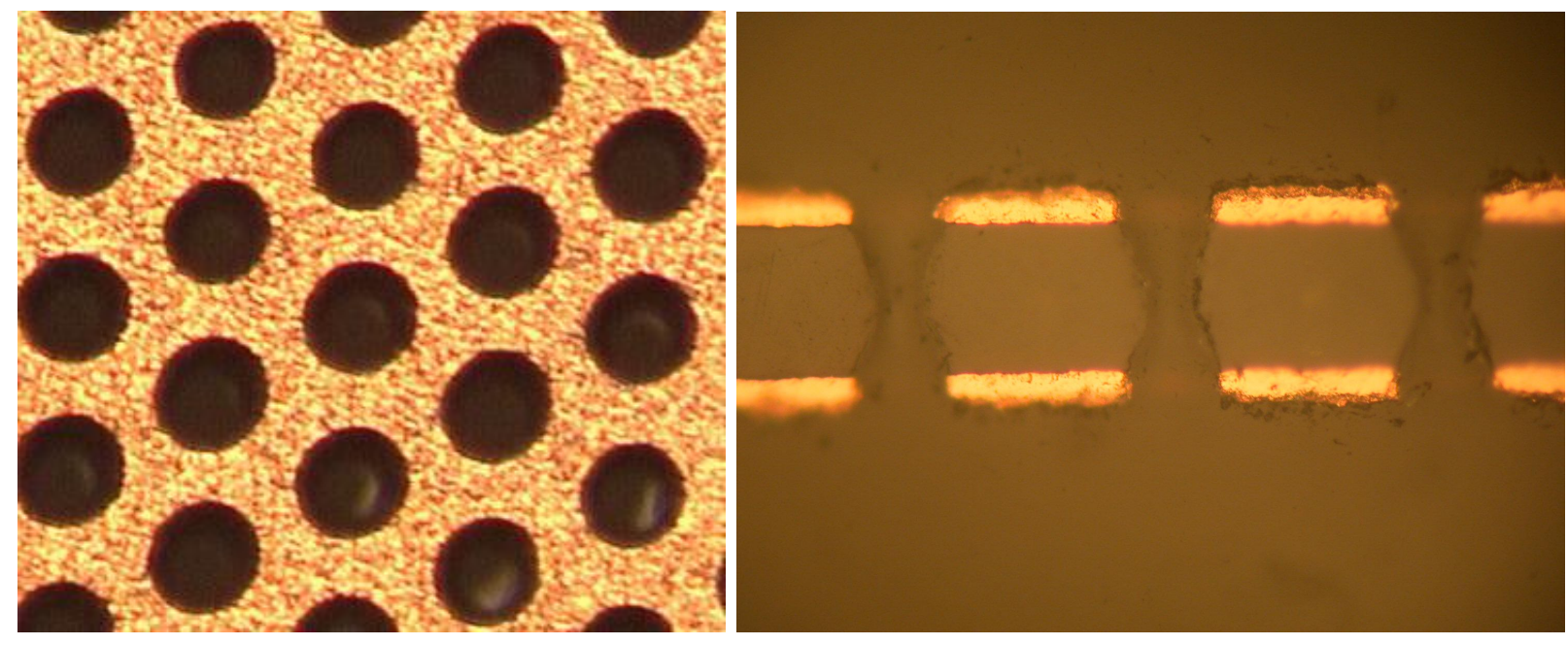

Figure 1. (Left) Magnified surface image of the laser etched GEM with pitch of $50 \mu \mathrm{m}$ and hole diameter of $30 \mu \mathrm{m}$. (Right) Cross section of the GEM with thickness of $50 \mu \mathrm{m}$.

We are interested in applying the GEMs to cosmic X-ray polarimeters in which GEMs are combined with fine pixel array detectors. ${ }^{7-10}$ Based on the photoelectric effect, polarization of the incident X-ray is obtained by determining the emission angle of the photoelectron, which is correlated with the electric field vector of the $\mathrm{X}$-ray. The key to increasing analyzing power is to accurately trace the photoelectron path, which is only about $1 \mathrm{~mm}$ even in neon at atmospheric pressure for $5 \mathrm{keV}$ X-ray,${ }^{11}$ though there is no sense in making pixels smaller than the lateral diffusion of drift electrons. If we carefully chose a gas and a depth of the drift region in order to extract the highest possible modulation, the pixel size is found to be $50-100 \mu \mathrm{m} .^{12}$

It is, however, difficult to produce a stable, high-gain GEM with a pitch less than $100 \mu \mathrm{m}$ for $50 \mu \mathrm{m}$-thick foil using the standard chemical etching technique. Therefore, we have pursued another technique for drilling the polyimide. ${ }^{13}$ The laser etching technique is the most promising method to produce fine pitch GEMs. Recently, we have produced the GEMs by using $\mathrm{CO}_{2}$ laser etching. ${ }^{14}$ The laser etching was originally employed by $\mathrm{J}$. Benlloch et al. ${ }^{15}$ and some other efforts were reported..$^{9,16,17}$

When we fabricate the fine-pitch GEMs, thinner foil is better for drilling polyimide properly. However, thicker foil is better for high-gain GEMs. Our goal is, therefore, to produce fine-pitch GEMs with thick-foil stably. In this paper, we present current status of our GEM productions and properties of the GEMs.

\section{PRODUCTION OF FINE-PITCH AND THICK-FOIL GEMS}

\subsection{Fine-pitch GEMs}

So far, the finest structure GEM we have fabricated has $30 \mu \mathrm{m}$-diameter holes on a $50 \mu \mathrm{m}$-pitch. The base plate is $50 \mu \mathrm{m}$-thick polyimide sandwiched by two $5 \mu \mathrm{m}$-thick copper layers. Fig. 1 shows the surface and cross section image of the $50 \mu \mathrm{m}$-pitch GEM drilled by the $\mathrm{CO}_{2}$ laser. The hole is slightly double conical shape with small taper angle of less than 10 degrees. The taper is slightly shallower than that of CERN standard GEMs.

We used six different types of GEMs in this work, listed in Table 1. The so-called "standard GEM" fabricated by the CERN-GDD group ${ }^{18}$ using chemical etching with a pitch of $140 \mu \mathrm{m}$ and a hole diameter of $70 \mu \mathrm{m}$, is called CERN-140. The same geometry as CERN-140 made with $\mathrm{CO}_{2}$ laser etching is designated as RIKEN-140. The $\mathrm{CO}_{2}$ laser-etched GEM fabricated by us with a pitch of $50 \mu \mathrm{m}$ and a hole diameter of $30 \mu \mathrm{m}$ is called RIKEN-50. All of the above GEMs have $5 \mu \mathrm{m}$-thick copper electrodes and a $50 \mu \mathrm{m}$-thick polyimide layer. Each GEM has $30 \times 30 \mathrm{~mm}^{2}$ active area. The other three types of GEMs are mentioned in the next subsection. 


\subsection{Thick-foil GEMs}

We have produced the GEMs with liquid crystal polymer (LCP) as an insulator instead of polyimide. One of the shortcomings of the polyimide is its moisture absorption. The absorption of moisture during the GEM production process causes transformation of polyimide layer into wavy shape. To avoid the transformation, we have employed LCP. While typical moisture absorption is $1.5 \%$ for polyimide in the atmosphere at $25{ }^{\circ} \mathrm{C}$ with $50 \%$ humidity, the moisture absorption is less than $0.04 \%$ for LCP. ${ }^{19}$ In addition, contamination of water vapor from the detector materials causes serious problem, which lowers the gas gain, for gas counters. We can reduce the contamination if we use LCP.

In this work we used three different types of GEMs produced with LCP. One of them has $50 \mu \mathrm{m}$-thick foil with hole diameter of $70 \mu \mathrm{m}$ and pitch of $140 \mu \mathrm{m}$, called RIKEN-140LCP. Second has the same hole diameter and pitch, but the thickness is $100 \mu \mathrm{m}$, called RIKEN-140LCP-100T. The last one has also the same hole diameter and pitch, but an $8 \mu \mathrm{m}$-thick copper layer is sandwiched by two $50 \mu \mathrm{m}$-thick LCP layers and two $8 \mu \mathrm{m}$-thick copper layers are plated on both side of the LCP-Cu-LCP layer. The last one called RIKEN-140LCP-2L. The cross sections of RIKEN-140LCP, RIKEN-140LCP-100T, and RIKEN-140LCP-2L are shown in Fig. 2. Each GEM has also $30 \times 30 \mathrm{~mm}^{2}$ active area. All of the RIKEN GEMs listed in the Table 1 were designed at RIKEN and fabricated by Scienergy Co. Ltd. *

Table 1. List of GEM foils examined in this study. All dimensions are given in $\mu \mathrm{m}$. The fourth column shows the thickness of only the insulator layers. The copper electrodes have $5 \mu \mathrm{m}$ in thickness for copper plated polyimide and 8 $\mu \mathrm{m}$ for the copper plated liquid crystal polymer.

\begin{tabular}{cccccc}
\hline name & pitch & hole dia. & thickness & insulator & etching method \\
\hline \hline CERN-140 & 140 & 70 & 50 & polyimide & chemical \\
\hline RIKEN-140 & 140 & 70 & 50 & polyimide & $\mathrm{CO}_{2}$ laser \\
RIKEN-50 & 50 & 30 & 50 & polyimide & $\mathrm{CO}_{2}$ laser \\
\hline RIKEN-140LCP & 140 & 70 & 50 & liquid crystal polymer & $\mathrm{CO}_{2}$ laser \\
RIKEN-140LCP-100T & 140 & 70 & 100 & liquid crystal polymer & $\mathrm{CO}_{2}$ laser \\
RIKEN-140LCP-2L & 140 & 70 & $50 \times 2$ layer & liquid crystal polymer & $\mathrm{CO}_{2}$ laser \\
\hline
\end{tabular}

\section{PROPERTY OF THE GEMS}

\subsection{Experimental Setup}

Fig. 3 shows a schematic view of the GEM test setup. It consists of a drift plane, GEM foils, and $3 \times 3$ readout pads with each area of $10 \times 10 \mathrm{~mm}^{2}$. Only the central readout pad was read out in the study and the other pads were connected to ground. The drift plane is $15 \mu \mathrm{m}$-thick aluminum foil with active area of $30 \times 30 \mathrm{~mm}^{2}$. The drift plane, GEMs, and readout pads are placed in a gas filled chamber. The spacing between the drift plane and the upper GEM (i.e. the spacing of target region) is $5.5 \mathrm{~mm}$, and that between the lower GEM and readout pad is $1.0 \mathrm{~mm}$. The electric field in the drift region is $\mathrm{E}_{d}=2.5 \mathrm{kV} / \mathrm{cm}$, and $\mathrm{E}_{i}=4 \sim 5 \mathrm{kV} / \mathrm{cm}$ for the induction region. A GEM is often used as a preamplifier to the other GEM to reduce discharge probability and ion feedback. ${ }^{20}$ When we studied the GEMs in the multi layer configuration, the spacing among GEMs was $2.0 \mathrm{~mm}$ with electric field of $\mathrm{E}_{t} \sim 2 \mathrm{kV} / \mathrm{cm}$. We did not take care to align GEMs in this study because transverse diffusion in the gap exceeds the pitch of the GEM holes. The high voltage is supplied via a chain of $10 \mathrm{M} \Omega$ resistors, and a $2 \mathrm{M} \Omega$ protection resistor is added in series with each GEM electrode.

Charge signals from the readout pads are fed into a charge sensitive preamplifier and shaper module (AmpTek A225). The amplified and shaped voltage signals are fed into the custom-made main amplifier. This module has a discriminator and gate generator for data acquisition system control. The amplified signals are fed into

${ }^{*}$ Contact address of Scienergy Co. Ltd. is info@scienergy.jp. 

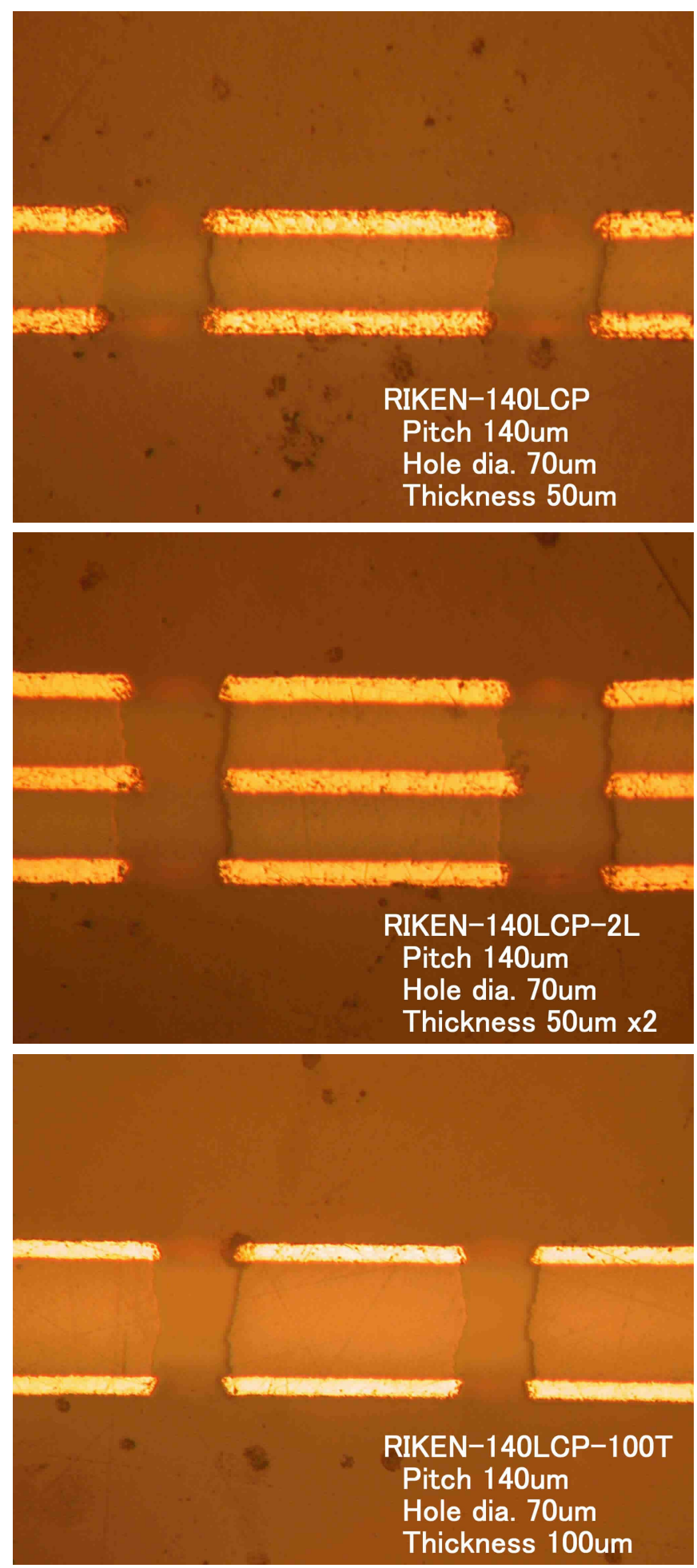

Figure 2. (Upper) The cross section of the RIKEN-140LCP GEM with pitch of $140 \mu \mathrm{m}$ and hole diameter of $70 \mu \mathrm{m}$. The insulator between the copper layers is LCP with thickness of $50 \mu \mathrm{m}$. (Middle) The cross section of RIKEN-140LCP-2L. There is the $8 \mu \mathrm{m}$-thick copper layer in the middle of the insulator layer. (Lower) The cross section of the RIKEN140LCP-100T GEM with $100 \mu$ m-thick LCP laser. 


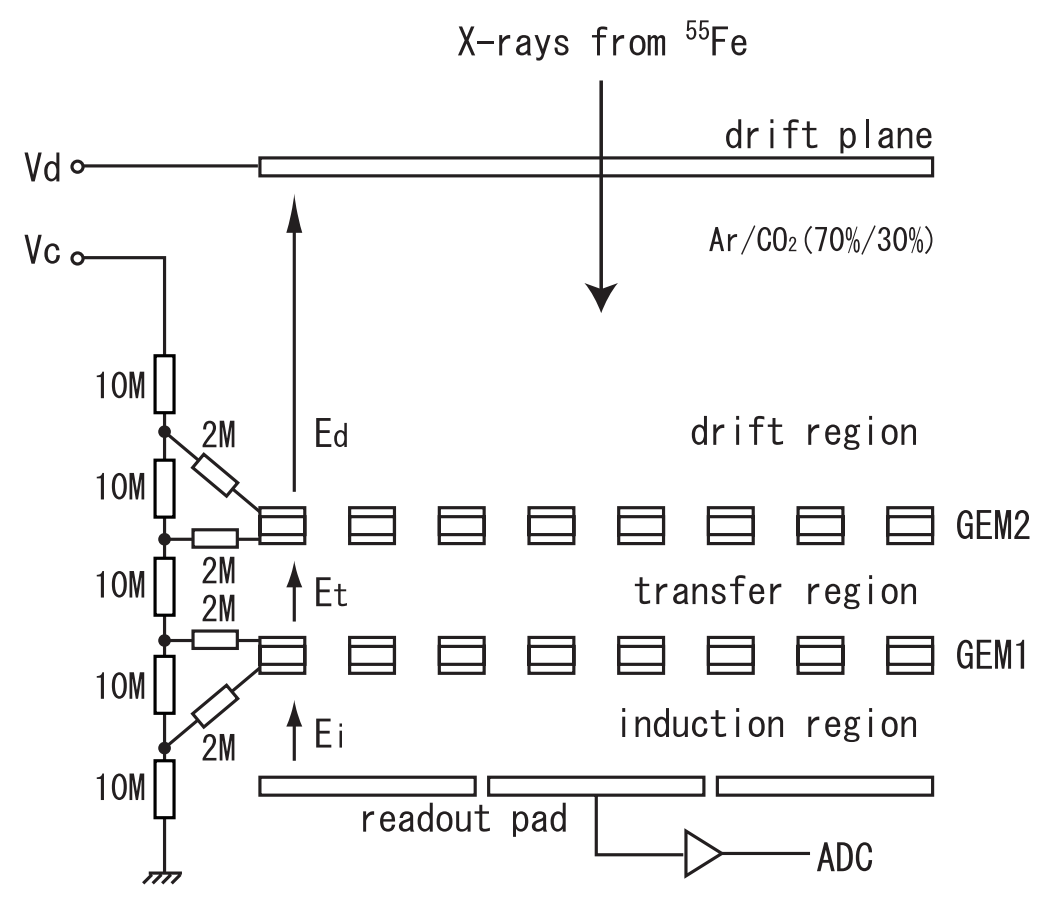

Figure 3. A schematic view of the experimental setup.

CAMAC peak-hold ADC (LeCroy 2259B) controlled by a PC. To make a calibration curve between amount of input charge and ADC channel, a well-defined rectangular wave from a research pulser (ORTEC model 448) was fed into the preamplifier through a $2 \mathrm{pF}$ capacitor.

During the test, we flowed a mixture of $70 \%$ argon and $30 \%$ carbon dioxide by volume through the chamber. The $\mathrm{CO}_{2}$ sense as a quencher. The primary reason we selected this gas mixture was to easily compare our results to other experiments; many GEM studies have been done with this gas mixture. We did not add any gases to prevent discharge, aging effects, etc. in this study.

\subsection{Gain of the GEMs}

We have measured electron gain as a function of applied voltage between the two copper electrodes of a GEM. Since it is difficult to measure the real GEM gain in the amplification channel, we define effective gain $\left(\mathrm{G}_{e f f}\right)$ as the ratio of detected charge to input one, and use this definition of gain throughout this paper. In this study, the effective gain is measured with $5.9 \mathrm{keV}$ X-ray irradiation from a ${ }^{55} \mathrm{Fe}$ radioactive source. Note that the effects of charge losses during the electron transfer in gas, GEMs and readout pads are merged into the $\mathrm{G}_{e f f}$ in this study.

The main peak in the ${ }^{55} \mathrm{Fe}$ spectrum corresponding to the $5.9 \mathrm{keV}$ X-ray is fitted with a Gaussian to measure the central value $\left(\mathrm{S}_{\text {mean }}\right)$ of the peak. The effective gain is found as:

$$
G_{\text {eff }}=\text { Const } \times \frac{S_{\text {mean }}}{q_{e} n_{e}}
$$

where $q_{e}$ is the electron charge and $n_{e}$ is the number of electron-ion pairs created by the absorption of $5.9 \mathrm{keV}$ X-ray. Mean value of $n_{e}$ is 212 for a mixture of $70 \%$ argon and $30 \%$ carbon dioxide. ${ }^{21}$ The constant value is found from the calibration curve between amount of input charge and ADC channel. 


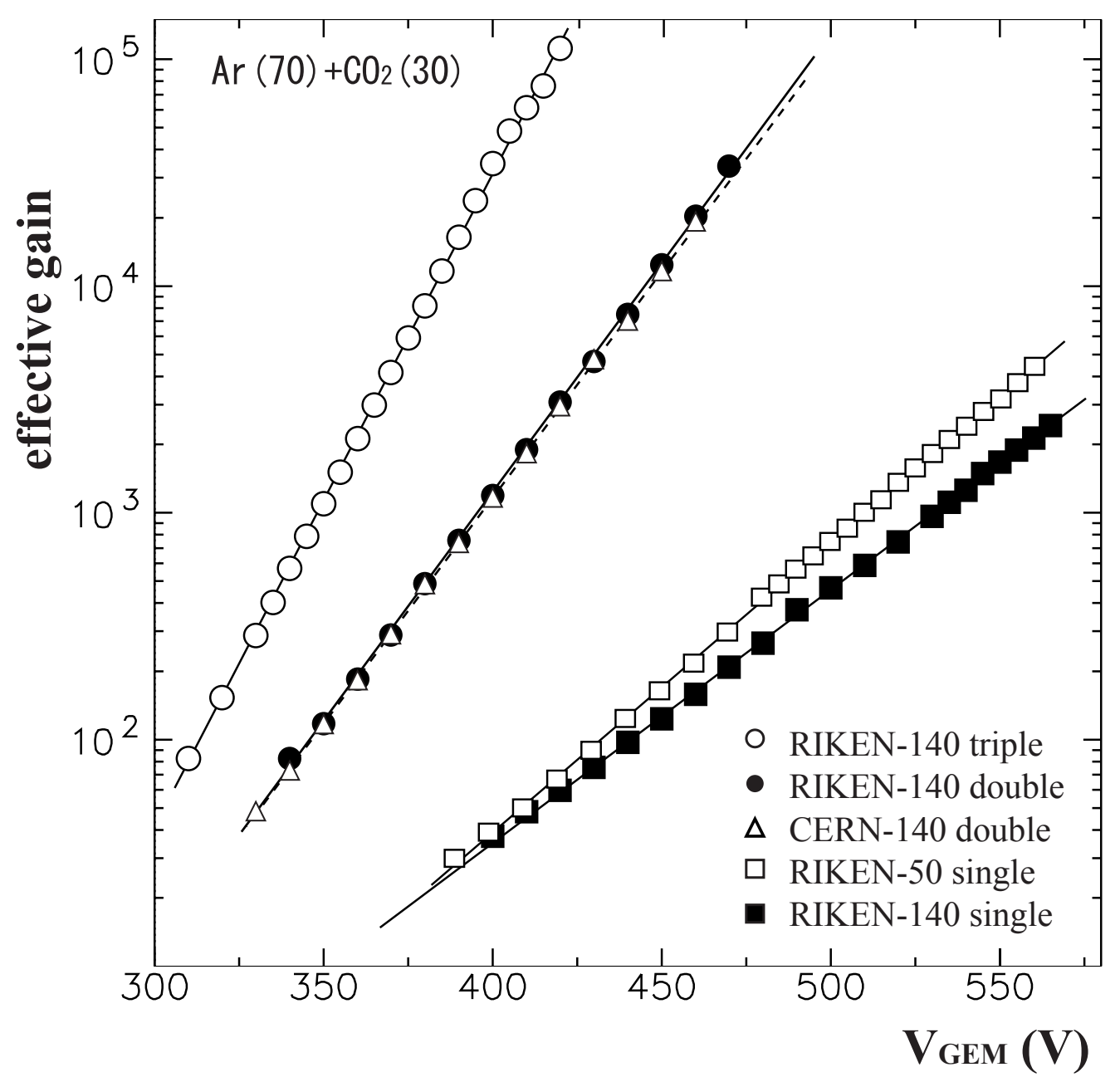

Figure 4. Effective gains of single, double and triple RIKEN-140 GEMs as a function of applied voltage between GEM electrodes. The gain of single finest-pitch GEM, RIKEN-50, is also plotted. The gain of double CERN-140 GEMs is plotted in the figure for comparison.

The effective gain of single, double, and triple RIKEN-140 GEMs are shown in Fig. 4. The effective gain of the double GEM is roughly the square of the gain of the single GEM, and that of triple GEM is roughly the cubic of the gain of the single GEM. The result of double layers of CERN-140 GEMs is also shown in the figure for comparison. The agreement between the gain curves of the CERN-140 and RIKEN-140 GEMs indicates that the gain is determined only by the geometry of the hole size and pitch. The effective gain of single RIKEN-50, which is the finest-pitch GEM we have fabricated, is also shown in Fig. 4. The gain is slightly higher than that of RIKEN-140 at the same applying voltage. This is probably due to the smaller hole diameter.

Fig. 5 shows gain properties of single layer of thick-foil RIKEN-140LCP-100T and RIKEN-140LCP-2L, which have $100 \mu \mathrm{m}$ thickness. For comparison, gain properties of double layer of thin-foil RIKEN-140LCP's are shown in the figure. The total thickness of GEMs are the same among them. However, single layer of the thick-foil GEMs have larger gain than double layer of the thin-foil GEMs, i.e. the gain curve is steeper. An effective gas gain of $10^{4}$ is achieved with RIKEN-140LCP-100T and RIKEN-140LCP-2L at the applied voltage of $350 \mathrm{~V}$ per 50 $\mu \mathrm{m}$ of thickness. The trend, thicker-foil GEM has larger gain than thin-foil one, is predicted from a simulation study done by Bouianov et al. ${ }^{22}$ Another important character of the thick-foil GEMs is the micro-discharge voltage is considerably higher than that of the thin-foil GEMs. It is around $\mathrm{G}_{e f f}=1-2 \times 10^{4}$ for the thick-foil 


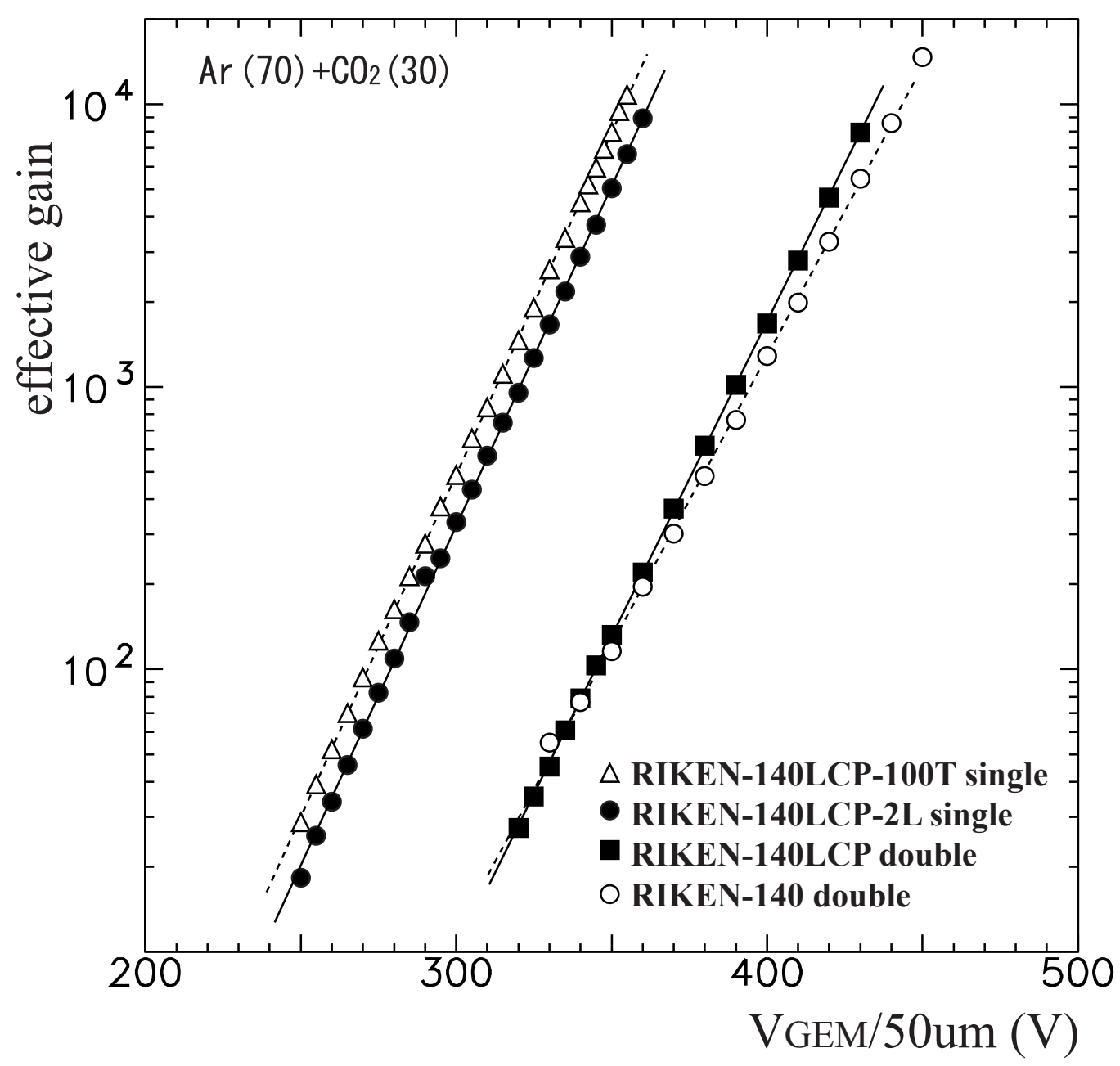

Figure 5. Figure captions are used to describe the figure and help the reader understand it's significance. The caption should be centered underneath the figure and set in 9-point font. It is preferable for figures and tables to be placed at the top or bottom of the page. LaTeX tends to adhere to this standard.

GEMs, whereas it is around $\mathrm{G}_{e f f}=2-3 \times 10^{3}$ for single layer of RIKEN-140LCP.

Since we have used LCP as an insulator layer instead of polyimide (PI) for the first time, we have compared the gain property between them. The effective gain of double layer of RIKEN-140, which has Cu-PI-Cu foil, are shown in the Fig. 5. The gain curve of RIKEN-140LCP has steeper than that of RIKEN-140. We have no idea why the gradient of the gain curves between them at this moment. One of the possible reasons is the difference comes from the geometry of hole edge. While there is about $10^{\circ}$ taper for the PI GEMs, there is almost cylindrical for the LCP GEMs. (See the right panel of Fig 1 and the top panel of Fig. 2.)

\subsection{Electric Field of the GEMs}

In Fig. 5, RIKEN-140LCP-100T has slightly higher gain than RIKEN-140LCP-2L. To study the difference, we have simulated the electric fields of them by using MAXWELL3D field simulator. ${ }^{23}$ Since we have not calculated electron multiplication in the simulation with GARFIELD ${ }^{24}$ we just mention a trend of gain qualitatively. Fig. 6 shows an equipotential surface map of RIKEN-140LCP-2L and RIKEN-140LCP-100T. One can see the equipotential surface is lined-up by the middle copper layer of RIKEN-140LCP-2L, i.e. the middle metal layer works as a field shaper. On the other hand, there is no such constrained condition for RIKEN-140LCP-100T. 

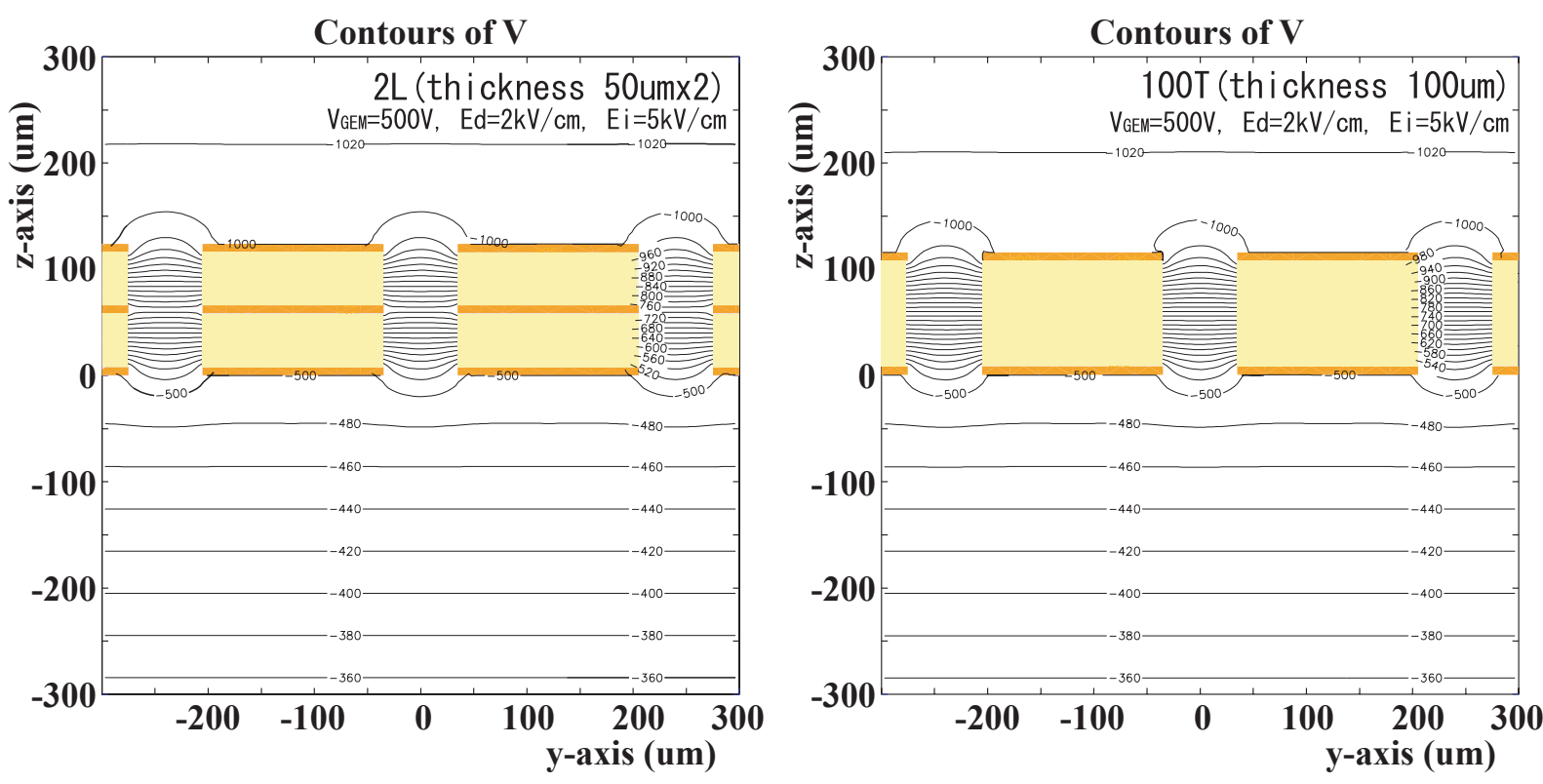

Figure 6. Equipotential surface for RIKEN-140LCP-2L (left) and RIKEN-140LCP-100T (right).

Fig. 7 shows the strength of electric field along the line perpendicular to the GEM foil for RIKEN-140LCP-2L and RIKEN-140LCP-100T. The electric field of RIKEN-140LCP-2L becomes small around the copper electrode in the middle of the layer. The effect is conspicuous along the line through the hole edge. This is why the effective gain of RIKEN-140LCP-2L is smaller than that of RIKEN-140LCP-100T. For the $100 \mu \mathrm{m}$-thick foil, the middle copper layer makes the effective gain worse. Since it shapes electric field in the amplification channels, the middle layer probably works better in the effective gain and prevents micro-discharge for thicker foils.

\section{SUMMARY AND FUTURE WORKS}

We have developed fine-pitch and thick-foil GEMs using laser etching technique for cosmic X-ray polarimeters. The minimum dimension of the GEM we have fabricated is $50 \mu \mathrm{m}$-pitch and $30 \mu \mathrm{m}$-diameter. The maximum thickness of the GEM is $100 \mu \mathrm{m}$.

We have measured the effective gain for $140 \mu \mathrm{m}$-pitch and $70 \mu \mathrm{m}$-diameter GEMs and $50 \mu \mathrm{m}$-pitch and 30 $\mu \mathrm{m}$-diameter GEMs. The $140 \mu \mathrm{m}$-pitch GEM was compared to the same structure GEM fabricated by CERN, and we found that the gain property is nearly the same. The effective gain of single layer of the $50 \mu \mathrm{m}$-pitch GEM is slightly larger than that of $140 \mu \mathrm{m}$-pitch GEM due to probably the smaller hole diameter. The gain reaches about 5000 at the voltage of $570 \mathrm{~V}$ between electrodes.

Liquid crystal polymer (LCP) has been employed for producing thicker-foil GEMs. A gas gain of $10^{4}$ is achieved with the $100 \mu \mathrm{m}$-thick GEMs at the applied voltage of $350 \mathrm{~V}$ per $50 \mu \mathrm{m}$ of thickness. On the other hand, the gain of $50 \mu \mathrm{m}$-thick GEM is $10^{2}$ at the same voltage. We have produced two different type of thick-foil GEMs. One of them has $100 \mu$ m-thick LCP layer sandwiched by two copper layers. The other has a middle copper layer sandwiched by two $50 \mu \mathrm{m}$-thick LCP layers and copper electrodes. The middle metal layer works as a field-shaper. The effective gain is, however, decreased slightly by the layer.

In the next production, we will fabricate thicker-foil GEMs with thickness of 150-200 $\mu \mathrm{m}$. we hope they have higher gas gain than $100 \mu$ m-thick GEM, and they probably keep voltage without discharge beyond effective gain $>10^{4}$. We also plan to fabricate $50 \mu \mathrm{m}$-pitch GEM with thickness of $100 \mu \mathrm{m}$, and apply it to our X-ray polarimeter. ${ }^{10}$ 

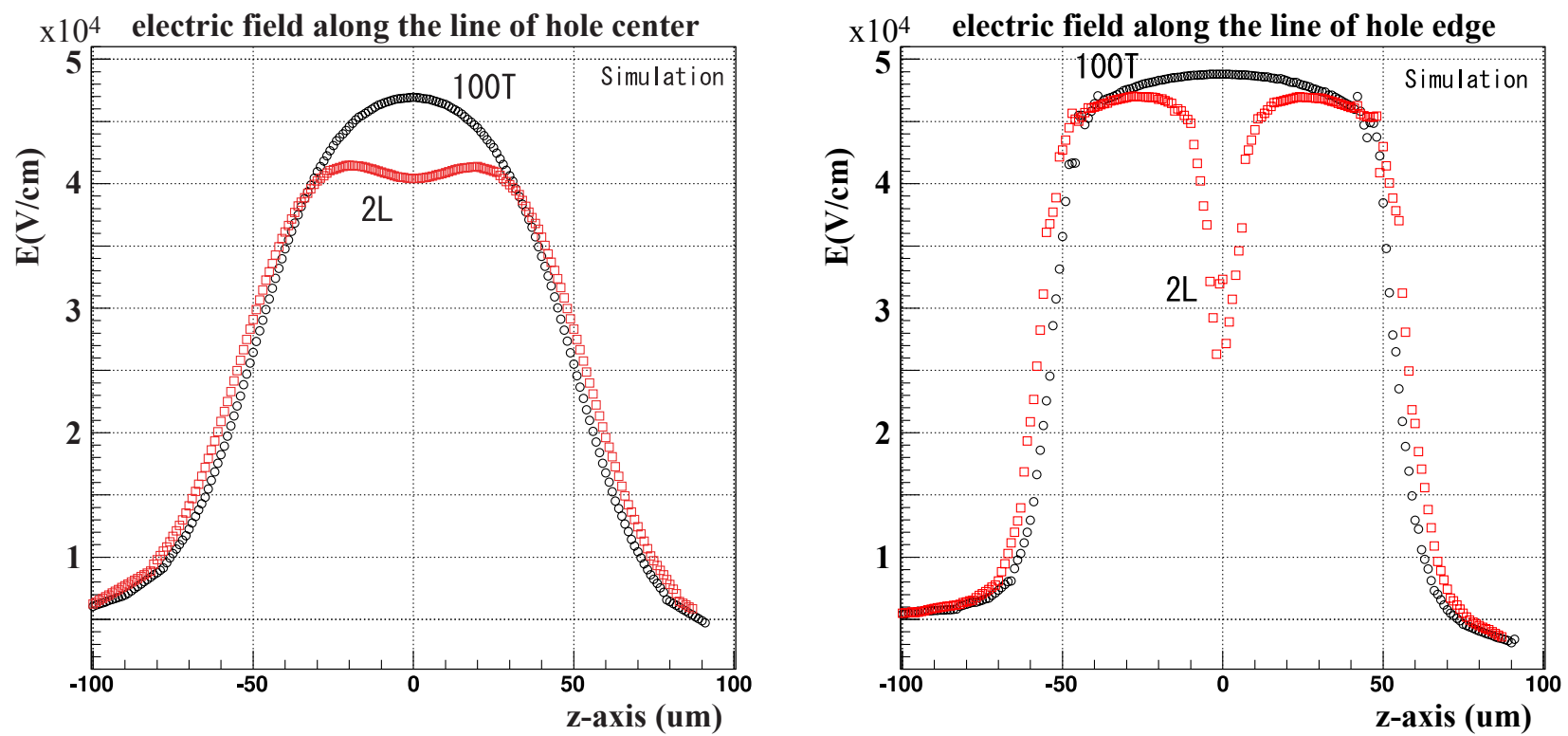

Figure 7. Electric field strength which a primary electron feels along the line perpendicular to the GEM surface and through the hole center (left), and along the line through the hole edge (right) for single layer of RIKEN-140LCP-2T and RIKEN-140LCP-100T. V

\section{REFERENCES}

1. F. Sauli, "Gem: A new concept for electron amplification in gas detectors," Nucl. Instrum. Meth. A386, pp. 531-534, 1997.

2. S. Bachmann et al., "Performance of gem detectors in high intensity particle beams," Nucl. Instrum. Meth. A470, pp. 548-561, 2001.

3. A. Buzulutskov et al., "The gem photomultiplier operated with noble gas mixtures," Nucl. Instrum. Meth. A443, pp. 164-180, 2000.

4. D. Mormann, A. Breskin, R. Chechik, and C. Shalem, "Operation principles and properties of the multi-gem gaseous photomultiplier with reflective photocathode," Nucl. Instrum. Meth. A530, pp. 258-274, 2004.

5. A. Breskin et al., "Ion-induced effects in gem and gem/mhsp gaseous photomultipliers for the uv and the visible spectral range," Nucl. Instrum. Meth. A553, pp. 46-52, 2005.

6. F. Sauli, "Gas detectors: Achievements and trends," Nucl. Instrum. Meth. A461, pp. 47-54, 2001.

7. E. Costa, P. Soffitta, R. Bellazzini, A. Brez, N. Lumb, and G. Spandre, "An efficient photoelectric x-ray polarimeter for the study of black holes and neutron stars," Nature 411, pp. 662-665, 2001.

8. R. Bellazzini, L. Baldini, E. Costa, L. Latronico, N. Omodei, P. Soffitta, and G. Spandre, "A photoelectric polarimeter based on a micropattern gas detector for x-ray astronomy," Nucl. Instrum. Meth. A510, pp. 176184, 2003.

9. J. K. Black, P. Deines-Jones, S. E. Ready, and R. A. Street, "X-ray polarimetry with an active-matrix pixel proportional counter," Nucl. Instrum. Meth. A513, pp. 639-643, 2003.

10. A. Hayato et al., "Development of cosmic x-ray polarimeter," Proc. SPIE 6266. (in this proceeding).

11. T. Tabata, R. Ito, and S. Okabe, "Generalized semiempirical equations for the extrapolated range of electrons," Nucl. Instrum. Meth. 103, pp. 85-91, 1972.

12. R. Bellazzini, F. Angelini, L. Baldini, A. Brez, E. Costa, et al., "X-ray polarimetry with a micro pattern gas detector with pixel read out," IEEE Trans. Nucl. Sci. NS-49, p. 1216, 2002.

13. M. Inuzuka, H. Hamagaki, K. Ozawa, T. Tamagawa, and T. Isobe, "Gas electron multiplier produced with the plasma etching method," Nucl. Instrum. Meth. A525, pp. 529-534, 2004. 
14. T. Tamagawa et al., "Development of gas electron multiplier foils with a laser etching technique," Nucl. Instrum. Meth. A, 2006. (in press).

15. J. Benlloch et al., "Development of the gas electron multiplier (gem)," IEEE Trans. Nucl. Sci. 45, pp. 234$243,1998$.

16. W. K. Pitts et al., "Development and operation of laser machined microwell detectors," Nucl. Instrum. Meth. A438, pp. 277-281, 1999.

17. H. S. Cho et al., "Gem: Performance and aging tests," IEEE Trans. Nucl. Sci. 46, pp. 306-311, 1999.

18. CERN Gas Detectors Development Group. http://gdd.web.cern.ch/GDD/.

19. Nippon Steel Chemical Co., Ltd. http://www.nscc.co.jp/english/materials/index.html.

20. A. Bondar, A. Buzulutskov, L. I. Shekhtman, and A. Vasiljev, "Study of ion feedback in multi-gem structures," Nucl. Instrum. Meth. A496, pp. 325-332, 2003.

21. F. Sauli CERN Yellow Report 77-09, 1997.

22. O. Bouianov, M. Bouianov, R. Orava, and V. Tikhonov, "Foil geometry effects on gem characteristics," Nucl. Instrum. Meth. A458, pp. 698-709, 2001.

23. Maxwell 3-D Field Simulator, User's Reference, Ansoft Corporation.

24. R. Veenhof. Garfield: A Drift-Chamber Simulation Program, Version 6.32. 